CARRAZZA, L.G. et al. Qualidade do sêmen em suínos submetidos ao estresse pelo calor:

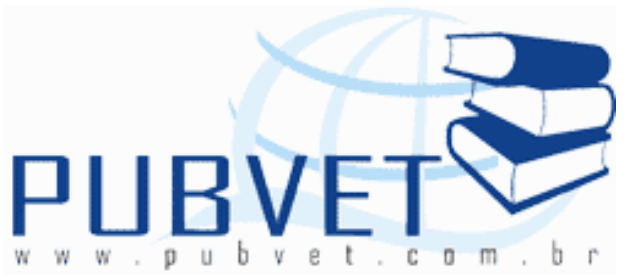

PUBVET, Publicações em Medicina Veterinária e Zootecnia.

\title{
Qualidade do sêmen em suínos submetidos ao estresse pelo calor: Revisão de literatura
}

Leonardo Gomes Carrazza ${ }^{1}$, Thaís Gomes Carrazza², Driele Schneidereit Sant'ana ${ }^{1}$, Millene Torres de Oliveira ${ }^{1}$, Luana Ribeiro Alves ${ }^{1}$

${ }^{1}$ Aluno de Mestrado do Programa de pós-graduação da Faculdade de Medicina Veterinária da Universidade Federal de Uberlândia - UFU.

${ }^{2}$ Médica Veterinária autônoma.

\section{Resumo}

Suínos são animais extremamente sensíveis a mudanças na temperatura ambiente, e reagem negativamente quando expostos a temperaturas ambiente elevadas, uma vez que possuem limitada capacidade de dissipação de calor. Esta revisão teve como objetivo abordar os principais efeitos deletérios de altas temperaturas ambientais sobre a capacidade reprodutiva de suínos machos e as alterações nutricionais utilizadas para reduzi-las. Nos machos reprodutores sob estresse pelo calor, ocorrem diminuições do volume seminal, da concentração espermática, motilidade espermática, além de ocorrer um maior aumento no número de espermatozóides anormais. Além das tradicionais alterações nas estruturas de construções e equipamentos, a alteração na nutrição de machos suínos afetados por estresse térmico tem mostrado resultados positivos, sobretudo a adição de alguns nutrientes na alimentação de animais como a Vitamina $E$, selênio, cromo, zinco e magnésio.

Palavras-chave: espermatozóide, motilidade, temperatura 
CARRAZZA, L.G. et al. Qualidade do sêmen em suínos submetidos ao estresse pelo calor: Revisão de literatura. PUBVET, Londrina, V. 5, N. 23, Ed. 170, Art. 1146, 2011.

\title{
Semen quality in pigs subjected to heat stress: Literature review
}

\begin{abstract}
Swines are extremely sensitive animals to ambient temperature changes, and react negatively when exposed to high ambient temperatures, since they have limited capacity for heat dissipation. This review aimed to address the main deleterious effects of high ambient temperatures on the reproductive capability of male pigs and nutritional changes used to reduce them. In breeding males under heat stress, there are decreases in semen volume, sperm concentration, sperm motility, and experience a greater increase in abnormal sperm. Besides the traditional changes in the structures of buildings and equipment, the change in the nutrition of male pigs affected by heat stress has shown positive results, especially the addition of some nutrients in food animals such as Vitamin E, selenium, chromium, zinc and magnesium.
\end{abstract}

Keywords: sperm, motility, temperature

\section{INTRODUÇÃO}

Em países tropicais os períodos de calor intenso são marcados por temperaturas expressivas que ocorrem principalmente durante o final da primavera, no verão e no início do outono, nos meses de outubro a março (INMET, 2004). Os suínos são susceptíveis a essas temperaturas, devido a sua limitada capacidade de eliminação de calor corporal, visto que apresentam uma espessa camada de tecido adiposo subcutâneo, limitada capacidade de perda de calor por sudorese e pelo reduzido número de glândulas sudoríparas presentes na pele (EINARSSON et al., 1996; DYCE et al., 1997). Como consequência, esses animais têm uma menor tolerância ao calor em relação a muitos outros animais domésticos e são susceptíveis à hipertermia quando expostos ao estresse pelo calor (WENTZ et al., 2001).

O estresse pode ser definido como uma reação do organismo a uma condição adversa do ambiente, onde ocorre uma tentativa de realizar a 
CARRAZZA, L.G. et al. Qualidade do sêmen em suínos submetidos ao estresse pelo calor: Revisão de literatura. PUBVET, Londrina, V. 5, N. 23, Ed. 170, Art. 1146, 2011.

termorregulação e manter a homeostasia (MACHADO FILH; HÖTZEL, 2000). Qualquer estímulo ambiental sobre um indivíduo que sobrecarregue seus sistemas de controle e reduza a sua adaptação, ou tenha potencial para isto, resulta em estresse (FRASER; BROOM, 1990).

Calor e umidade elevada podem resultar em estresse crônico, especialmente se acompanhado por uma ampla flutuação da temperatura, resultando em diminuição na ingestão de alimento e interferência na espermatogênese (KUNAVONGKRIT et al., 2005). Considera-se que os animais estão expostos a estresse térmico quando a temperatura ambiente estiver acima da zona de conforto térmico e energia for gasta para manter a temperatura corporal (BLACK et al., 1992).

Como temperatura de conforto, entende-se aquela na qual se torna dispensável qualquer atividade metabólica por parte do animal para aquecer ou esfriar o corpo, na qual o metabolismo animal é mínimo (OLIVEIRA et al., 2003). Para os machos a temperatura ideal, segundo Perdomo et al. (1985), situa-se entre 12 e $21^{\circ} \mathrm{C}$, sendo a temperatura crítica inferior igual a $12^{\circ} \mathrm{C}$ e a temperatura crítica superior igual a $26^{\circ} \mathrm{C}$.

Dentro da zona de conforto térmico, a energia da dieta é utilizada para crescimento, manutenção e atividade física. Abaixo desta temperatura, energia adicional é necessária para manter a homeotermia. Acima desta condição, todo calor produzido deve ser eliminado (COLLIN et al., 2001).

Embora os suínos procurem se adaptar às condições ambientais quando expostos a elevadas temperaturas pelo aumento rápido do cortisol, as concentrações deste hormônio retornam praticamente ao normal nas primeiras 72 h após a exposição. Porém, se a temperatura for alta durante o dia e baixa durante a noite, a adaptação torna-se difícil. Diferenças entre as temperaturas diurnas e noturnas maiores do que $10^{\circ} \mathrm{C}\left(25-35^{\circ} \mathrm{C}\right)$ e uma umidade maior do que $90 \%$ irão desencadear estresse maior nos animais que não conseguem se adaptar a tais mudanças. Desta forma, flutuações na temperatura durante o dia e à noite podem significar um fator desencadeante de estresse para os machos durante as estações quentes do ano (SEREN et al., 1988). 
CARRAZZA, L.G. et al. Qualidade do sêmen em suínos submetidos ao estresse pelo calor: Revisão de literatura. PUBVET, Londrina, V. 5, N. 23, Ed. 170, Art. 1146, 2011.

Deste modo, objetivou-se com esta revisão de literatura, a abordagem dos principais efeitos negativos de altas temperaturas ambientais sobre o desempenho reprodutivo de suínos machos, assim como estratégias para controle desses efeitos, através de alterações nutricionais.

\section{REVISÃO DE LITERATURA}

\subsection{Efeitos da temperatura ambiente sobre a reprodução de machos}

Os altos índices de temperatura e umidade no ambiente, principalmente durante os meses mais quentes do ano, podem afetar severamente a eficiência reprodutiva dos machos suínos (WETTEMANN et al., 1976).

A qualidade do sêmen pode ser afetada por diversos fatores como manejo, alojamento, sanidade, nutrição e clima (FLOWERS, 1997). Quando os animais encontram-se fora da zona de conforto térmico, a produção de calor aumenta e a taxa e a eficiência da utilização dos nutrientes são reduzidas (SILVA, 2000).

Diversos estudos realizados sob condições controladas de temperatura e umidade

demonstraram que as células espermáticas são afetadas pelos elementos climáticos e a gravidade das lesões depende da intensidade do estresse térmico e do período de exposição a eles (FLOWERS, 1997).

A produção de sêmen e o número total de espermatozóides no ejaculado dos cachaços são influenciados por todos aqueles fatores que possam alterar o processo de espermatogênese, como disponibilidade de alimento, idade, nutrição, efeitos ambientais, status sanitário e freqüência de montas ou colheitas, fatores estes que resultam em grandes variações das características do sêmen (MARTÍNEZ; CORCUERA, 2005).

Segundo Kunavongkrit et al. (2005), durante as estações mais quentes, o animal consome menos ração e o balanço nutricional de sua dieta pode ser 
CARRAZZA, L.G. et al. Qualidade do sêmen em suínos submetidos ao estresse pelo calor: Revisão de literatura. PUBVET, Londrina, V. 5, N. 23, Ed. 170, Art. 1146, 2011.

afetado. Cachaços com baixo consumo de proteína têm o início da ejaculação retardado e sua duração diminuída.

Cachaços submetidos a altas temperaturas ambientais têm sua fertilidade reduzida, pois, de acordo com Suriyasomboon (2005), tais condições podem causar uma degeneração testicular leve à moderada. Estes machos produzem ejaculados com menor concentração de espermatozóides, alta porcentagem de células espermáticas (espermatozóides) anormais e diminuição da motilidade e do volume espermático.

Segundo Waberski et al. (1994) a porcentagem de células espermáticas anormais é um parâmetro de avaliação seminal importante, pois provê informação sobre a eficiência da espermatogênese e facilita a seleção de machos para programas de inseminação artificial.

Sobestiansky et al. (1998) relataram que o alojamento de machos em ambientes com temperaturas acima de $30^{\circ} \mathrm{C}$ leva ao comprometimento da qualidade do sêmen, por afetar o processo de espermatogênese e reduzir a motilidade dos espermatozóides.

Em suas pesquisas, Sydenstricker (1993) afirmou que machos submetidos a altas temperaturas podem apresentar alterações na espermatogênese, devido ao aquecimento local dos testículos e ao desequilíbrio hormonal e metabólico, decorrentes do estresse, comprometendo a qualidade do sêmen.

Flowers (1997) afirmou que os efeitos negativos do estresse calórico sobre a

espermatogênese dos machos reprodutores suínos podem ser imediatos, severos e consistentes. Segundo o autor, o tempo mínimo de exposição e a temperatura crítica do ar nas quais a produção espermática é prejudicada, provavelmente variam de acordo com o local.

De acordo com Myer e Bucklin (2001), temperaturas acima de $29^{\circ} \mathrm{C}$ causam redução da produção e da qualidade dos espermatozóides. Valores próximos a este foram encontrados por Estienne (2000), cujas pesquisas têm indicado que o tempo mínimo de exposição e temperatura crítica do ar para 
CARRAZZA, L.G. et al. Qualidade do sêmen em suínos submetidos ao estresse pelo calor: Revisão de literatura. PUBVET, Londrina, V. 5, N. 23, Ed. 170, Art. 1146, 2011.

que a produção de células espermáticas seja afetada é de 72 horas e $29,4^{\circ} \mathrm{C}$, respectivamente.

Estienne (2000) relatou que um período de aproximadamente duas semanas é normalmente observado entre o estresse calórico agudo e as primeiras indicações na produção de espermatozóides anormais.

Suriyasomboon et al. (2004) analisando suínos criados sobre sistema convencional e evaporativo, encontraram significativo efeito sazonal sobre a porcentagem de espermatozóides morfologicamente normais e anormais com defeitos como gota citoplasmática proximal e anomalias na cabeça espermática. A temperatura tem significativo efeito negativo sobre os espermatozóides morfologicamente normais, aumentando o índice de gotas proximais tanto no sistema convencional quanto com sistema evaporativo. A umidade tem efeito negativo sobre as gotas proximais no sistema evaporativo.

Alterações na qualidade seminal de suínos selvagens nas diferentes estações do ano também foram observadas por Kozdrowski e Dubiel (2004). Os autores concluíram que os ejaculados de suínos selvagens não diferem dos ejaculados de suínos domésticos, e que o sêmen apresentou maior volume, concentração e número de espermatozóides no final do outono, sendo que a motilidade espermática foi menor no verão do que nas demais estações. Ainda de acordo com os autores, há uma expressiva correlação entre concentração espermática e volume da fração líquida do ejaculado. Tais resultados comprovam que a influência climática sobre a qualidade seminal é um fator inerente à espécie, não dependendo da linhagem genética dos animais.

A correlação entre 0 estresse causado pelas altas temperaturas ambientais e a diminuição de volume do sêmen e da concentração espermática pode ser atribuída à indução da produção excessiva de hormônios corticosteróides ocasionada pelo estresse segundo Kunavongkrit et al. (2005). De acordo com o autor, quando os suínos se encontram em instalações com temperaturas elevadas, o hormônio do estresse (cortisol) aumenta rapidamente, mas nas 72 horas seguintes retorna praticamente aos níveis normais. Isto indica a adaptação dos suínos às diversas temperaturas 
CARRAZZA, L.G. et al. Qualidade do sêmen em suínos submetidos ao estresse pelo calor: Revisão de literatura. PUBVET, Londrina, V. 5, N. 23, Ed. 170, Art. 1146, 2011.

ambientais, embora as temperaturas altas durante o dia e baixas durante a noite dificultem esta adaptação. Diferenças diárias de temperatura acima de $10^{\circ} \mathrm{C}$ e umidades acima de $90 \%$ irão gerar maior estresse nos cachaços mais sensíveis, que não poderão adaptar-se a essas mudanças.

De acordo com Suriyasomboon et al. (2004), o volume e a produção espermática total diminuem quando a temperatura e a umidade excedem $30^{\circ} \mathrm{C}$ e $40 \%$, respectivamente, no sistema convencional, e $27^{\circ} \mathrm{C}$ e $70 \%$, respectivamente, no sistema climatizado.

Kozdrowski e Dubiel (2004) observaram em sua pesquisa, que em cachaços adultos, a massa testicular e o nível de testosterona no sangue são significativamente maiores no inverno, quando comparados ao verão. Segundo Kunavongkrit et al. (2005), quando a concentração de testosterona é alta, o volume seminal, a concentração espermática e a viabilidade após congelamento seguido de descongelamento também são maiores, pois a testosterona é muito importante para a manutenção quantitativa da espermatogênese.

\subsection{Alternativas adotadas para melhorar a qualidade do sêmen de machos em estresse térmico}

De acordo com Bortolozzo et al. (2003), a produção espermática varia de acordo com o macho, raça, época do ano, meio ambiente, idade, nível nutricional e tamanho dos testículos. Nesse sentido, o fornecimento de condições ambientais adequadas para a plena produção espermática utilizando sistemas de ambiente controlado ou ventilação e aspersão são fundamentais para otimizar a produção espermática.

Além das alterações no ambiente, com instalações de equipamentos, visando melhorar a temperatura ambiental, causando resfriamento, tendências na nutrição também são observadas para tentar minimizar os efeitos deletérios do estresse térmico na fertilidade dos animais. 
CARRAZZA, L.G. et al. Qualidade do sêmen em suínos submetidos ao estresse pelo calor: Revisão de literatura. PUBVET, Londrina, V. 5, N. 23, Ed. 170, Art. 1146, 2011.

Com os avanços obtidos no melhoramento genético dos suínos, um plano nutricional mais elevado é necessário para atender as necessidades. Animais com plano nutricional mais elevado reagem mais drasticamente ao calor do que animais com plano nutricional mais baixo (FUQUAY, 1981).

Vários estudos mostram que o selênio (Se) e vitamina E (Vit. E) podem afetar positivamente a qualidade do sêmen suíno.

A suplementação de selênio reduz a degeneração dos túbulos seminíferos, a baixa motilidade e anomalias morfológicas em espermatozóidesem ratos e porcos (BRZEZINSKA-SLEBODZINSKA et al., 1995). Segundo os mesmo autores, a morfologia testicular e funções são afetados pela deficiência de Se e que o elemento é necessário para a biossíntese de testosterona e na formação e desenvolvimento normal dos espermatozóides.

A função do Vit. E é inteiramente associada às suas propriedades antioxidantes, e o sêmen suíno é extremamente sensível a danos peroxidativos devido ao alto conteúdo de ácidos graxos insaturados em fosfolipídios da membrana plasmática, além da relativa baixa capacidade antioxidante do plasma seminal. O dano estrutural aos espermatozóides ocorre quando devido a peroxidação lipídica, o que resulta em um declínio na motilidade espermática (BRZEZINSKA-SLEBODZINSKA et al., 1995).

Brzezinska-Slebodzinska et al. (1995) relataram um aumento na concentração de espermatozóides no sêmen de cachaços suplementados com Vit. E como um efeito associado às propriedades antioxidantes dessa vitamina.

Echeverria-Alonzo, et al. (2009), analisando a suplementação com Vitamina E e Selênio, em dietas de suínos machos em estação quente e fresca, relataram que adição desses nutrientes influenciou positivamente a concentração espermática, a motilidade espermática, a porcentagem de espermatozóides morfologicamente normais, além de reduzirem as anomalias de cabeça e a retenção de gotículas citoplasmáticas, nas duas estações, sendo mais relevante na estação quente, onde as alterações negativas no sêmen, tendem a ser maiores. 
CARRAZZA, L.G. et al. Qualidade do sêmen em suínos submetidos ao estresse pelo calor: Revisão de literatura. PUBVET, Londrina, V. 5, N. 23, Ed. 170, Art. 1146, 2011.

A nutrição dos machos reprodutores pode influenciar a quantidade de sêmen (número de espermatozóides e volume do ejaculado), especialmente em animais jovens e sob condições desfavoráveis de ambiente (HUGONIN, 2001).

Uma dieta contendo $14 \%$ de proteína ou nível de lisina de 0,7\% e 70\% de energia é recomendada para machos reprodutores e, quando reduzida a ingestão diária, a produção de sêmen será reduzida (FLOWERS, 1997).

No que diz respeito à dieta, deve-se considerar a importância dos minerais, principalmente aqueles necessários para a produção espermática e desenvolvimento testicular, em especial a importância dos microminerais orgânicos cobre, cromo, manganês, iodo, selênio, zinco e ferro, cuja suplementação leva ao aumento do volume do ejaculado e redução dos efeitos estressantes aos quais os animais são submetidos (MAHAN et al., 2002).

O zinco é um elemento mineral que apresenta importante função para os machos, pois desempenha papel vital na secreção de hormônios, especialmente aqueles relacionados ao crescimento, reprodução, imunocompetência e estresse, sendo essencial para o desenvolvimento sexual e espermatogênese (CLOSE, 2000), por sua influência sobre o desenvolvimento das células de Leydig e produção de esteróides a nível testicular (HESKETH, 1982). Desta forma, recomendam-se níveis de ao menos 100 mg/kg na ração de machos suínos (CARRIÓN; MEDEL, 2001).

Animais estressados alimentados normalmente manifestam sinais de deficiência de cromo, verificados por decréscimo na eficiência alimentar, aumento do hormônio do estresse e função imune comprometida. Várias formas de estresse aumentam a mobilização das reservas de cromo, sendo que em animais estressados demonstrou-se o aumento dos requerimentos de cromo. Pelo exposto, é necessária a suplementação de cromo para suínos em particular sob condições de estresse e doença (van HEUGTEN; SPEARS, 1997).

A suplementação dietética contendo magnésio também alivia os efeitos do estresse pela redução da concentração de cortisol plasmático, noradrenalina, adrenalina e dopamina, e a grande vantagem do magnésio 
CARRAZZA, L.G. et al. Qualidade do sêmen em suínos submetidos ao estresse pelo calor: Revisão de literatura. PUBVET, Londrina, V. 5, N. 23, Ed. 170, Art. 1146, 2011.

orgânico em relação ao inorgânico é verificada pelo aumento da biodisponibilidade deste composto (D'SOUZA; MULLAN, 1998).

\section{CONSIDERAÇÕES FINAIS}

Os suínos, com o aumento da idade, tornam-se bastante sensíveis a altas temperaturas ambientais, uma vez que não possuem mecanismos eficientes de eliminação do calor corporal. Esse estresse causado por altas temperaturas afeta, sobretudo machos em fase de reprodução, onde a qualidade do sêmen destes animais tende a diminuir pois ocorre diminuição do volume do ejaculado, da concentração e mobilidade espermática e aumento do número de espermatozóides anormais,

Além das tradicionais alterações nas estruturas de construções e equipamentos, a alteração na nutrição de machos suínos afetados por estresse térmico tem mostrado resultados positivos, sobretudo a adição de alguns nutrientes na alimentação de animais como a Vitamina $E$, selênio, cromo, zinco e magnésio.

\section{REFERÊNCIAS BIBLIOGRÁFICAS}

BLACK, J. L.; MULLAN, B. P.; LORSCHY, M. L.; GILES, L. R. Lactation in the sow during heat stress. Livestock Production Science, v. 35, p. 153-170, 1992.

BORTOLOZZO, F. P.; DALLANORA, D.; BERNARDI, M. L.; BENNEMANN, P. E.; WENTZ, I. Técnicas associadas à inseminação artificial no suíno que visam a redução do número de espermatozóides necessários por fêmea ao ano. Revista Brasileira de Reprodução Animal. v. 27, n. 2, p. 133-139, 2003.

BRZEZINSKA-SLEBODZINSKA, E. Antioxidant status of dairy cows supplemented prepartum with vitamin E and selenium. Journal of Dairy Science, v. 77, p. 3087-3095, 1994.

CARRIÓN, D.; MEDEL, P. Interacción Nutrición Reproducción en Ganado Porcino. In: XVII Curso de Especialización FEDNA: AVANCES EN NUTRICIÓN Y ALIMENTACIÓN ANIMAL, 2001, Madrid, Espanha, p. 27-70.

CLOSE, W. H. Producing pigs without antibiotic growth promoters. Advances

in Pork Production, v. 11, p. 47, 2000. 
COLLIN, A.; VAN MILGEN, J.; DUBOIS, S.; NOBLET, J. Effect of high temperature on feeding behaviour and heat production in group-housed young pigs. British Journal of Nutrition, $v$. 86, p. 63-70, 2001.

DYCE, K. M.; SACK, W. O; WENSING, C. J. G. Anatomia veterinária. 2. ed. Rio de Janeiro: Guanabara Koogan, 1997. 663 p.

D'SOUZA, D. N.; MULLAN, B. P. Dietary nutrient supplements improve meat quality. In: BIOTECHNOLOGY IN THE FEED INDUSTRY, 1998, Nothingham.

UK. Proceedings of Alltech's 14th Annual Symposium, Nothingham, United Kingdom, 1998, 11p.

ECHEVERRIA-ALONZO, S.; SANTOS-RICALDE, R. ; CENTURION-CASTRO, F.; AKE-LOPEZ, R.; ALFARO-GAMBOA, M.; RODRIGUEZ-BUENFIL. Effects of Dietary Selenium and Vitamin E on Semen Quality and Sperm Morphology of Young Boars During Warm and Fresh Season.

Journal of Animal and Veterinary Advances, v. 8, n. 11, p. 2311-2317, 2009.

EINARSSON, S.; MADEJ, A.; TSUMA, V. The influence of stress on early pregnancy in the pig. Animal Reproduction Science, v. 42, p. 165-172, 1996.

ESTIENNE, M. J. Keep boars cool during summer. Livestock Update. Virginia Cooperative Extension. 2000. Disponível em: <http://www.ext.vt.edu/news/periodicals/livestock/aps00_07/aps- 0246.html>. Acesso em: 28 nov. 2010.

FLOWERS, W. L. Management of boars for efficient semen production. Journal of Reproduction and Fertility Supplement. n. 52 (in press). 1997.

FRASER, A. F.; BROOM, D. M. Farm animal behaviour and welfare. Wallingford: $C A B$ International, 1990. 448p.

FUQUAY, J. W. Heat stress as it affects animal production. Journal of Animal Science, v. 52, n. 1, p. $164-172,1981$.

HESKETH, J. E. Effects of dietary zinc deficiency on Leydig cell ultrastructure in the boar. Journal of Comparative Pathology, v. 92, p. 239-247, 1982.

HUGONIN, L. Avanços tecnológicos na nutrição de machos reprodutores suínos. In: Seminário Nacionalde Desenvolvimento da Suinocultura, 9., 2001, Gramado, RS. Anais... Concórdia: EMBRAPASuínos e Aves, 2001, p. 98. Disponível em:

<http://docsagencia.cnptia.embrapa.br/suino/anais/anais0104_hugonin.pdf>. Acesso em: 26 nov. 2010.

INMET. Instituto Nacional de Meteorologia. Disponível em: <http://www.inmet.gov.br/> . Acesso em: 28 nov. 2010.

KOZDROWSKI, R.; DUBIEL, A. The effect of season on the properties of wild boar (Sus scrofa L.) semen. Animal Reproduction Science. v. 80, p. 281-289. 2004.

KUNAVONGKRIT, A.; SURIYASOMBOON, A.; LUNDEHEIM, N.; HEARD, T. W.; EINARSSON, S. Management and sperm production of boars under differing environmental conditions.

Theriogenology, v. 63, p. 657-667, 2005.

MACHADO FILHO, L. C. P; HÖTZEL, M. J. Bem estar dos suínos. In: Seminário Internacional de Suinocultura, 5, 2000, São Paulo, SP. Anais... Concórdia: EMBRAPA Suínos e Aves, 2000. p. 70-82. 
MAHAN, D.; ZAWADZKI, J.; GUERREROR. Mineral metabolism and boar fertility: observations from Latin America to Europe. In: BIOTECHNOLOGY IN THEFEED INDUSTRY, 2002, Nothingham, UK. Proceedings of Alltech's 18th Annual Symposium, Nothingham, United Kingdom. p. 407-414. Disponível em:

<http://www.cababstractsplus.org/abstracts/Abstract.aspx?AcNo=20063209860 >. Acesso em: 28 nov. 2010.

MARTÍNEZ, P. C.; CORCUERA, B. D. U. Análise de Sêmen: Importância nos Resultados Reprodutivos. Suínos \& Cia, n. 13, p. 11-17. 2005.

MYER, R.; BUCKLIN, R. Influence of hot-humid environment on growth performance and reproduction of swine. AN107. Animal Science Department, Florida Cooperative Extension Service, Institute of Food and Agricultural Sciences, University of Florida. 2001.

OLIVEIRA, P. A. V.; PAULO, R. M.; TINÔCO, I. F. F. Efeito da temperatura no desempenho zootécnico de suínos em crescimento e terminação nos sistemas de camas sobrepostas e piso concretado. In: Congresso Brasileiro de Veterinários Especialistas em Suínos, 10, 2003, Concórdia, SC. Anais... Concórdia: EMBRAPA Suínos e Aves, 2003. p. 401.

PERDOMO, C. C.; KOZEN, E. A.; SOBESTIANSKY, J.; SILVA, A. P.; CORREA, N. I. Considerações sobre edificações para suínos. In: Curso de Atualização sobre a Produção de Suínos, 4, 1985, Concórdia, SC. Anais... Concórdia: EMBRAPA Suínos e Aves. 1985.

SEREN, E.; MATTIOLI, M.; RENSIS, F. Effect of high temperatures on LH and cortisol secretion in ovariectomized sows. In: International Congress on Animal Reproduction, 11, 1988, Dublin. Proceedings ... Dublin: ICAR, 1988, p. 417.

SILVA, R. G. Introdução à Bioclimatologia Animal. São Paulo: Nobel, 2000. 286 p.

SOBESTIANSKY, J; MORÉS, N.; BARCELLOS, D. E. S.; OLIVEIRA, S. J.; CARVALHO, L. F. O.; MORENO, A. M.; ROEHE, P. M. et al. Clínica e Patología Suína. 2a ed. Goiania: J. Sobestiansky. 464 p. 1998.

SYDENSTRICKER, K. V. Análise de Lanternim em Edificações para Suínos através de Modelos em Escala. 1993. 77f. Dissertação (Mestrado) - Faculdade de Engenharia Agrícola, Universidade Estadual de Campinas, Campinas.

SURIYASOMBOON, A.; LUNDEHEIM, N.; KUNAVONGKRIT, A.; EINARSSON, S. Effect of temperature and humidity on sperm production in Duroc boars under different housing systems in Thailand. Livestock Production Science, v. 89, p. 19-31. 2004.

SURIYASOMBOON, A. Herd Investigations on Sperm Production in Boars, and Sow Fertility under Tropical Conditions. 2005. 52f. Tese (Doutorado) - Faculdade de Medicina Veterinária e Ciência Animal, Departamento de Ciências Clínicas, Swedish University of Agricultural Sciences. Uppsala.

VAN HEUGTEN, E.; SPEARS, J. W. Immune response and growth of stressed weanling pigs fed diets supplemented with organic or inorganic forms of chromium. Journal of Animal Science. v. 75, p. 409-416, 1997.

WABERSKI, D.; MEDING, S.; DIRKSEN, G.; WEITZE, K. F.; LEIDING, C.; HAHN, R. Fertility of long-term-stored boar semen: Influence of extender (Androhep and Kiev), storage time and plasma droplets in the semen. Animal Reproduction Science, v. 36, p. 145-151, 1994. 
WENTZ, I.; BORTOLOZZO, F. P.; BRANDT, G.; HECK, A.; BONNEMANN, P. E.; GUIDONI, A. L.; UEMOTO, D. A. Ahipertermia durante o estro pode afetar o desempenho reprodutivo de fêmeas suínas. Ciência Rural, v. 31. p. 651-656. 2001.

WETTEMANN, R. P.; WELLS, M. E.; OMTVEDT, I.T.; POPE, C. E.; TURMAN, E. J. Influence of elevated ambient temperature on reproductive performance of boars. Journal od Animal Science, v. 42, p. 664-669, 1976. 